\title{
Cancer Vaccine Therapy Using Tumor Endothelial Cells as Antigens Suppresses Solid Tumor Growth and Metastasis
}

\author{
Tetsuya Nomura, ${ }^{a, b}$ Keiichi Hirata, ${ }^{a}$ Takuto Shimaoka, ${ }^{a}$ Makie Yamakawa, ${ }^{b}$ Naoya Koizumi, ${ }^{b}$ \\ Ryo Suzuki, ${ }^{a}$ Kazuo Maruyama, ${ }^{a}$ and Naoki Utoguchi*,a,b \\ ${ }^{a}$ Faculty of Pharma-Sciences, Teikyo University; 2-11-1 Kaga, Itabashi-ku, Tokyo 173-8605, Japan: and \\ ${ }^{b}$ Department of Pharmaceutics and Biopharmaceutics, Showa Pharmaceutical University; \\ 3-3165 Higashitamagawagakuen, Machida, Tokyo 194-8543, Japan. \\ Received February 15, 2017; accepted June 26, 2017
}

\begin{abstract}
Tumor angiogenesis plays an important role in tumor growth and metastasis, with tumor cells requiring nutrients and oxygen via blood flow for their proliferation. In comparison, angiogenesis also occurs under normal physiological conditions, such as wound healing and in the formation of the corpus luteum. Herein, we report on the development of a novel dendritic cell (DC) vaccine therapy using tumor endothelial cells (TECs) derived from tumor vessels as tumor antigens. After density gradient centrifugation and the detection of angiotensin-converting enzyme activities, a TEC-rich fraction was separated from solid tumor tissues. Prophylactic or therapeutic immunization using DCs pulsed with TECs as vaccine antigens significantly suppressed solid tumor growth in a Colon-26 colorectal adenocarcinoma tumor-bearing mouse model, compared with the use of tumor cells as DC vaccine antigens. Tumor tissues showed reduced angiogenesis. However, vaccination using DCs pulsed with TECs did not inhibit physiological angiogenesis as evidenced by a wound healing assay. Additionally, in a B16/BL6 mouse melanoma lung metastasis model, DC vaccination using TECs derived not only from the same tumor tissue but from a different type of tumor also suppressed metastasis. These results thus show that cancer vaccine therapy targeting TECs is an effective therapy against angiogenesis in several types of cancer, but does not affect normal blood vessel growth.
\end{abstract}

Key words tumor endothelial cell; angiogenesis; dendritic cell; vaccine; metastasis

Endothelial cells that enable tumor angiogenesis are recruited from neighboring, pre-existing capillaries ${ }^{1,2)}$ by tumor angiogenesis mediators, such as vascular endothelial growth factor (VEGF) ${ }^{3)}$ fibroblast growth factors, ${ }^{4)}$ and transforming growth factor, ${ }^{5)}$ in the process of tumor progression or metastasis. ${ }^{6,7)}$ Newly produced blood vessels, which are constructed by tumor endothelial cells (TECs), provide oxygen and nutrients, and remove metabolic waste from tumor cells; tumor cell proliferation also depends on new blood vessel formation. ${ }^{6,8)}$ In addition, TECs in different types of cancers express common, tumor-associated antigens (TAAs), since the tumor's new blood vessels are constructed from endothelial cells of the host. In this regard, it is expected that the inhibition of angiogenesis in tumor tissue will effectively induce tumor regression for several types of cancer, compared with the direct inhibition of tumor cells themselves. In fact, several antiangiogenic agents, such as the VEGF receptor and tyrosine kinase inhibitor, sunitinib, ${ }^{9)}$ and the anti-VEGF antibody, bevacizum$a b,{ }^{10)}$ are commonly applied in clinical practice today since they tend to show a survival advantage for patients. However, angiogenesis also occurs during the processes of wound healing and corpus luteum formation, ${ }^{11)}$ with the implication that the clinical application of antiangiogenic agents in cancer patients may result in damage to normal blood vessels, ${ }^{12)}$ in addition to those of the tumor. Therefore, the selective inhibition of tumor angiogenesis is expected to result in the development of cancer therapy with fewer side effects.

Recent implementation of a dendritic cell (DC) vaccine therapy approach to cancer treatment has been employed, instead of more established treatments such as surgery, chemotherapy, and radiotherapy. ${ }^{13)}$ DCs are antigen-presenting cells that can express epitope peptides derived from TAAs on the cell surface via major histocompatibility complex class I and II molecules, and which prime naive T cells..$^{14,15)}$ In such therapy, specific cytotoxic T-lymphocytes (CTLs) for TAAs are induced by vaccination with TAA-loaded DCs to effectively induce anti-tumor immunity to tumor tissues. ${ }^{16)}$ In fact, several TAAs, such as Wilms tumor 1 (WT1), New York esophageal squamous cell carcinoma 1 (NY-ESO-1) and mucin 1 (MUC1), have been identified as derived from tumor cells and this has led to extensive clinical research with DCs. ${ }^{17-19)}$

In this study, we have attempted to develop a DC vaccine therapy using TECs isolated from solid tumor tissue as TAAs. However, the methods used to isolate TECs from solid tumor tissue are not fully developed since a specific marker for TECs has not, as yet, been conclusively identified. Therefore, in a bid to develop an appropriate TEC isolation method, a TEC-rich fraction was separated from solid tumors in mice by density-gradient centrifugation, and assessed on the basis of angiotensin-converting enzyme (ACE) activity, ${ }^{20}$ ) which is abundantly found at the surface of endothelial cells, where it catalyzes the conversion of the inactive decapeptide, angiotensin I, to angiotensin II and converts bradykinin to inactive fragments. The anti-tumor effect of vaccination with DCs loaded with a TEC-rich extract was evaluated in both tumorbearing and lung metastasis mouse models. Moreover, the effect of DC vaccination with TECs on normal blood vessels was observed in a wound healing mouse model.

\section{MATERIALS AND METHODS}

Animals $\mathrm{BALB} / \mathrm{c}$ and $\mathrm{C} 57 \mathrm{BL} / 6$ mice were purchased 
from Sankyo Labo Service Corporation, Inc. (Tokyo, Japan). All experiments were conducted according to the institutional ethical guidelines for animal experimentation of Teikyo University (Tokyo, Japan).

Cells Colon-26 murine colorectal adenocarcinoma cells were provided by Dr. S. Unezaki (University of Tokyo Medical, Tokyo). Colon-26 cells were maintained at $37^{\circ} \mathrm{C}$ in $5 \%$ $\mathrm{CO}_{2}$ in RPMI 1640 medium supplemented with $10 \%$ fetal bovine serum (FBS), streptomycin $(0.5 \mathrm{mg} / \mathrm{mL})$, and penicillin $\mathrm{G}$ (500 U/mL). B16/BL6 (B16) mouse melanoma cells were purchased from the American Type Culture Collection (ATCC, Manassas, VA, U.S.A.). B16 cells were maintained at $37^{\circ} \mathrm{C}$ in $5 \% \mathrm{CO}_{2}$ in minimum essential medium (MEM) supplemented with $10 \%$ FBS, streptomycin $(0.5 \mathrm{mg} / \mathrm{mL})$, and penicillin $\mathrm{G}$ $(500 \mathrm{U} / \mathrm{mL})$. Mouse hepatic sinusoidal endothelial cells (HSE) were provided by Dr. I. Saiki (University of Toyama, Japan) and maintained at $37^{\circ} \mathrm{C}$ in $5 \% \mathrm{CO}_{2}$ in Dulbecco's modified Eagle's medium (DMEM)/F-12 medium supplemented with $10 \%$ FBS, streptomycin $(0.5 \mathrm{mg} / \mathrm{mL})$, Endothelial Mitogen (Biomedical Technologies, Inc., Stoughton, MA, U.S.A.; $100 \mu \mathrm{g} / \mathrm{mL})$, and penicillin $\mathrm{G}(500 \mathrm{U} / \mathrm{mL})$.

Tumor Models For solid tumor model studies, Colon-26 or B16 cells $\left(1.0 \times 10^{6}\right.$ cells) were injected subcutaneously (s.c.) into the flanks of $\mathrm{BALB} / \mathrm{c}$ or $\mathrm{C} 57 \mathrm{BL} / 6$ mice, respectively. Tumor volume was calculated by the use of the modified ellipsoid formula, $1 / 2$ (length $\times$ width $\left.^{2}\right)$. For lung metastatic model studies, B16 cells $\left(1.0 \times 10^{5}\right.$ cells $)$ were injected intravenously (i.v.) into the tail vein of $\mathrm{C} 57 \mathrm{BL} / 6$ mice. After two weeks, mice were sacrificed and lungs fixed in $10 \%$ neutral buffered formalin. The number of B16 metastasis colonies on the lung surface was counted with a stereoscopic dissecting microscope.

TEC Isolation from Solid Tumors by Density Gradient Centrifugation Colon-26 or B16 tumor tissues were removed when tumor volume reached $4000 \mathrm{~mm}^{3}$. Tumor tissues were digested with $0.75 \%$ collagenase $(\mathrm{pH} 7.3$; Wako Pure Chemical Industries, Ltd., Osaka, Japan) containing $0.5 \%$ bovine serum albumin (BSA) and $0.0072 \%$ deoxyribonuclease (DNase) (Wako Pure Chemical Industries, Ltd.) in Hank's Balanced Salt Solution (HBSS; Sigma-Aldrich, St. Louis, MO, U.S.A.) for $1 \mathrm{~h}$ at $37^{\circ} \mathrm{C}$. The cell suspension was washed by centrifugation at $1000 \mathrm{rpm}$ for $4 \mathrm{~min}$ in MEM containing $10 \%$ FBS. The cell pellet was then resuspended in MEM containing $10 \%$ FBS and gently layered on the top of an established $28 \%$ (30\% for B16 tumor cells) Percoll (Sigma-Aldrich) density gradient. Gradient tubes were centrifuged at $2000 \mathrm{rpm}$ in a swing rotor for $10 \mathrm{~min}$, and the gradient was fractionated from the bottom of the Percoll gradient at $1 \mathrm{~mL}$ intervals.

ACE Activity Each cell suspension was washed twice with HBSS by centrifugation at $4000 \mathrm{rpm}$ for $5 \mathrm{~min}$; cell pellets were then resuspended in borate buffer $(\mathrm{pH} 8.3)$ and sonicated. The ACE activity of each extracted cell fraction was determined by a previously described method, ${ }^{20)}$ and was expressed in terms of activity per $\mu \mathrm{g}$ protein of cell fraction. Briefly, each cell extract containing ACE activity was incubated for $1 \mathrm{~h}$ at $37^{\circ} \mathrm{C}$ with $4.5 \mathrm{~mm}$ Benzoylglycyl-Lhistidyl-L-leucine (BzGly-His-Leu) $\cdot \mathrm{H}_{2} \mathrm{O}$ (Peptide Institute, Inc., Osaka, Japan) as a substrate, and then $0.5 \%$ fluorescent $o$-phthalaldehyde (Wako Pure Chemical Industries, Ltd.) in $\mathrm{MeOH}$ and $1 \mathrm{~N} \mathrm{NaOH}$ were added for $10 \mathrm{~min}$ at room temperature. After $30 \mathrm{~min}$ incubation in $3 \mathrm{~N} \mathrm{HCl}$, fluorescence inten- sity was measured using an excitation wavelength of $360 \mathrm{~nm}$ and an emission wavelength of $500 \mathrm{~nm}$ in a microplate reader. Protein amounts were measured by Micro BCA Protein Assay Reagent Kit (Thermo Scientific, Rockford, IL, U.S.A.), with BSA used as a standard protein.

CD34 Expression on the Surface of Isolated TECs Cell suspensions $\left(1.0 \times 10^{6}\right.$ cells $)$ were stained with fluorescein isothiocyanate (FITC) anti-mouse CD34 antibody (MEC14.7; Santa Cruz Biotechnology, Santa Cruz, CA, U.S.A.; $10 \mu \mathrm{g} / \mathrm{mL}$ ) for $1 \mathrm{~h}$ at room temperature. After staining, cells were washed twice by centrifugation at $1500 \mathrm{rpm}$ for $5 \mathrm{~min}$ in phosphate buffered saline (PBS). CD34 expression on the cell surface was analyzed using a FACS Calibur flow cytometer (Becton Dickinson, Franklin Lakes, NJ, U.S.A.) and CellQuest software (BD Biosciences, San Jose, CA, U.S.A.).

Extraction of Antigenic Proteins Each cell was resuspended in PBS, and lysed by several cycles of freezing and thawing, followed by centrifugation. The supernatants were used as antigenic proteins.

Generation of Mouse Bone Marrow-Derived DCs DCs were generated from mouse bone marrow cells according to previously described methods. ${ }^{21)}$ Briefly, bone marrow cells were isolated from $\mathrm{BALB} / \mathrm{c}$ or $\mathrm{C} 57 \mathrm{BL} / 6$ mice and cultured in RPMI 1640 supplemented with 10\% FBS, 2-mercaptoethanol (2-ME; Invitrogen, Life Technologies, Carlsbad, CA, U.S.A.; $50 \mu \mathrm{M}$ ), mouse granulocyte macrophage colonystimulating factor (mGM-CSF; Peprotech Inc, Rocky Hill, NJ, U.S.A.; $20 \mu \mathrm{g} / \mathrm{mL})$, penicillin $\mathrm{G}(100 \mathrm{U} / \mathrm{mL})$, and streptomycin $(100 \mu \mathrm{g} / \mathrm{mL})$ at $37^{\circ} \mathrm{C}$ in $5 \% \mathrm{CO}_{2}$. The culture medium was changed every three days, and non-adherent cells were collected as immature DCs after $10 \mathrm{~d}$ of culture. Phenotypic characterization of the DCs performed by flow cytometry using DC markers (CD11c, CD40, CD80 and CD86) and MHC molecules (classes I and II) indicated that the cells after incubation were DC marker-positive (data not shown).

Immunization of Mice with Antigen-Loaded DCs DCs $\left(2.0 \times 10^{7}\right.$ cells $\left./ 8 \mathrm{~mL}\right)$ were pulsed with antigenic proteins $(600 \mu \mathrm{g} / 2 \mathrm{~mL})$ using Lipofectin (Invitrogen, Life Technologies; $100 \mu \mathrm{g} / 100 \mu \mathrm{L})$ in $100 \mathrm{~cm}^{2}$ dishes. After $5 \mathrm{~h}$ incubation at $37^{\circ} \mathrm{C}$ in $5 \% \mathrm{CO}_{2}$, the DCs were washed three times with PBS, and DCs $\left(1.0 \times 10^{6}\right.$ cells $\left./ 100 \mu \mathrm{L}\right)$ were then injected twice intradermally into the backs of mice, with a one-week interval.

Mouse Dorsal Air Sac Model for Angiogenesis The mouse dorsal air sac model was constructed according to a previously reported method. ${ }^{22}$ Briefly, the chamber was prepared by covering both sides of a Millipore ring with a $0.45 \mu \mathrm{m}$ Millipore filter. Seven days after the final DC immunization, the chamber, which was filled with a suspension of Colon-26 tumor cells $\left(1 \times 10^{6}\right.$ cells $\left./ 150 \mu \mathrm{L}\right)$, was embedded s.c. into the backs of mice. Five days after chamber implantation, mice were sacrificed and chambers removed. The angiogenic response was evaluated by the formation of tumor blood vessels. The chamber was observed using a stereoscopic microscope (Olympus, Tokyo, Japan). The number of newly formed serpiginous blood vessels within the area attached by a chamber ring was counted by averaging four random fields per specimen.

Histopathological Analysis For the histopathological analysis of tumor tissues $40 \mathrm{~d}$ after tumor implantation, mice were sacrificed and Colon-26 solid tumors removed. Tumor tissue was fixed with a $4 \%$ neutral formalin buffer, and then 
tissues were embedded in paraffin. Paraffin blocks were sectioned in a sliding microtome (Leica, Bensheim, Germany). After tissue sections were deparaffinized and dehydrated with alcohol and xylene, tissues were stained with hematoxylineosin and observed by stereoscopic microscope. The number of new blood vessels in the intratumoral section was counted by averaging four random fields per specimen.

Wound Healing Assay Wound healing was evaluated as previously described. ${ }^{23)}$ Briefly, $7 \mathrm{~d}$ after the final immunization, six circular wounds, each $5 \mathrm{~mm}$ in diameter, were made on the backs of BALB/c mice. The number of wound sites was counted every day, and the number of days until wound closure was also measured.

Statistical Analysis The statistical significance of differential findings between experimental groups and controls was determined by one-way ANOVA or Student's $t$-test, and con- sidered significant if a $p$ value was $<0.01,0.005$ or 0.001 . Data are presented as mean \pm standard error of the mean (S.E.M.). All experiments were performed at least twice.

\section{RESULTS}

Characterization of ACE Activity and CD34 Expression in Cell Fractions Containing TECs Isolated from Solid Tumors To isolate TECs from a Colon-26 solid tumor, a cell suspension was prepared by collagenase digestion and cells separated by Percoll density gradient centrifugation. Each cell suspension was fractionated from the bottom of the gradient, and the ACE activity of each cell fraction was measured (Fig. 1). The ACE activity per $\mu \mathrm{g}$ protein of cells in fractions No. 5 to 7 was much higher than that of cells in other fractions (Fig. 1A). In particular, the ACE activities of cells in fractions No.

A

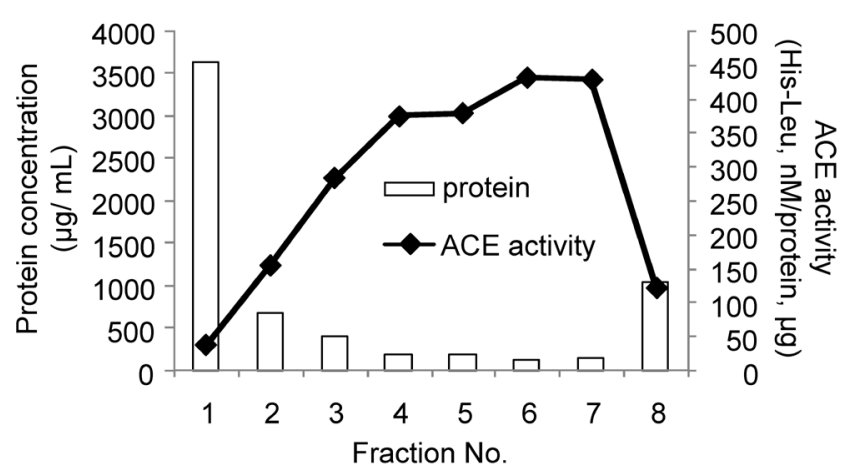

B

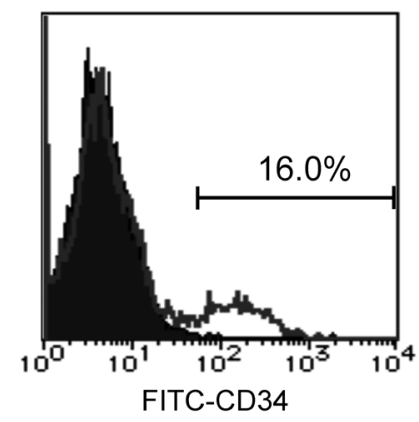

No. 1

D

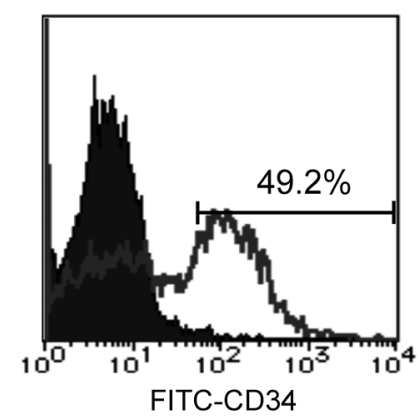

No. 6
C

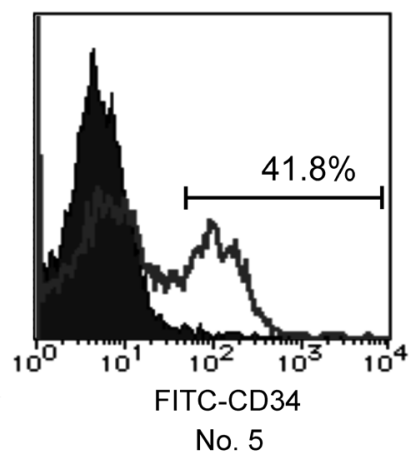

E

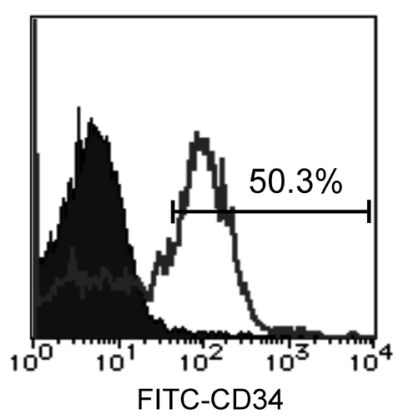

No. 7

Fig. 1. Characterization of the TEC-Rich Fraction Isolated from a Colon-26-Derived Solid Tumor

(A), ACE activities per $\mu \mathrm{g}$ protein of each fraction containing cells separated, using $28 \%$ Percoll density gradient centrifugation, from solid tumor tissues removed $14 \mathrm{~d}$ after $1 \times 10^{6}$ Colon-26 cells were implanted s.c. in the flanks of mice. (B-E), CD34 expression on the surface of each fraction's cells as determined by flow cytometric analysis. Unstained cells (filled area). Anti-CD34 stained cells (unfilled area). Experiments were performed twice. 
6 and 7 were more than six times higher compared with that of fraction No. 1 .

In addition, CD34 expression on the membrane surface of each fraction's cells was measured by flow cytometric analysis. As with ACE activity, CD34 expression of cells in fractions No. 5 to $7(41.8-50.3 \%)$ was also higher compared with that of cells in fraction No. $1(16.0 \%)$ (Figs. 1B-E).

As noted above, many TECs were detected in fractions No. 5 to 7 . Cells in fractions No. 5 to 7 which contained more than 40\% CD34-positive cells were collected as TEC fractions. Cells in fraction No. 1 were collected as Low ACE fraction. For TECs derived from B16 tumor tissues, these were observed to be abundant within fractions No. 6 to 8 (data not shown). Thus TEC fractions were separated by density gradient centrifugation and identified on the basis of their high ACE activity and CD34 expression.

DC Vaccination Targeting TECs Inhibited Tumor Growth Effectivity To evaluate the efficacy of tumor vaccine therapy targeting TECs, DC vaccination using separated TEC-rich fractions as TAAs were performed in a tumor-bearing mouse model (Fig. 2). Firstly, the anti-tumor effects of a DC vaccine targeting TECs were compared with that of a DC vaccine targeting tumor cells themselves (Fig. 2A). Prophylactic vaccination using Colon-26 protein cell extract-pulsed DCs (Colon-26/DC) inhibited tumor growth compared with vaccination with DCs only ( $p<0.01 v s$. Non/DC; Fig. 2A). In contrast, vaccination using TECs isolated from Colon-26 solid tumors to pulse DCs (TEC/DC) significantly suppressed tumor progression in colon cancer in a more dominant manner than not only the Non/DC but also Colon-26/DC groups $(p<0.001$ or $p<0.01 v s$. Non/DC or Colon-26/DC, respectively; Fig. 2A).

Next, to clarify that the anti-tumor effects of vaccination with isolated TECs were not induced by a liquid factor secreted by the solid tumor in vivo, the inhibitory effect of TEC/DC vaccination on tumor growth was compared with solid tumor cell protein extract-pulsed DCs (STC/DC) and protein cell extract fractions with low ACE activity-pulsed DCs (Low $\mathrm{ACE} / \mathrm{DC}$ ) in a prophylactic model (Fig. 2B). In STC/DC mice, tumor growth was suppressed slightly compared to that of Non/DC mice. However, Low ACE/DC-treated mice did not show any anti-tumor effects. In contrast, TEC/DCtreated mice showed a significant inhibition of tumor growth $(p<0.001$ or $p<0.01 v s$. Non/DC or Low ACE/DC, respectively). In addition, for the therapeutic protocol, TEC/DC vaccination also significantly inhibited increases in tumor volume $(p<0.001 v s$. Non/DC or STC/DC, $)$, whereas tumor growth in STC/DC-treated mice was not suppressed (Fig. 2C). In all experiments, Non/DC vaccination hardly inhibited tumor growth, in common with untreated (data not shown).

Thus vaccination of DCs pulsed with TECs isolated from Colon-26 solid tumors inhibited tumor progression in colon cancer, both in prophylactic and therapeutic models, and this effect was not dependent on a factor secreted by tumor cells.

DC Vaccination Targeting TECs Inhibited Formation of Tumor Blood Vessels To evaluate whether vaccination by DCs pulsed with isolated TEC extracts could inhibit neovascularization in Colon-26 solid tumors, the formation of blood vessels by solid tumors in mouse skin was observed using dorsal air sac chambers (Fig. 3). In Non/DC-treated mice, many serpiginous vessels were noted in mouse tissue (Fig. 3B). However, tumor-induced neovascularization was
A

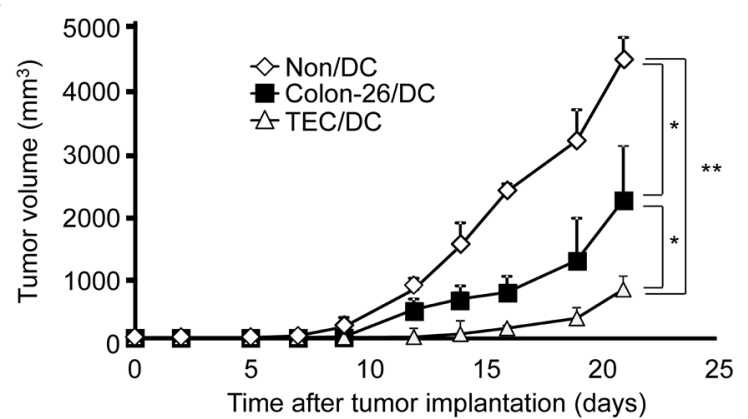

B

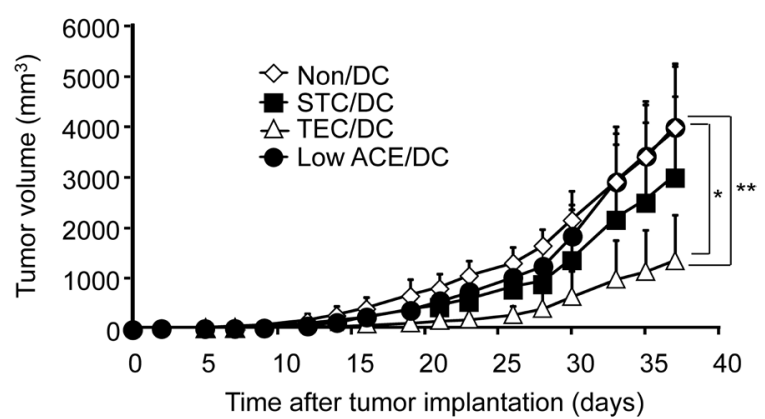

C

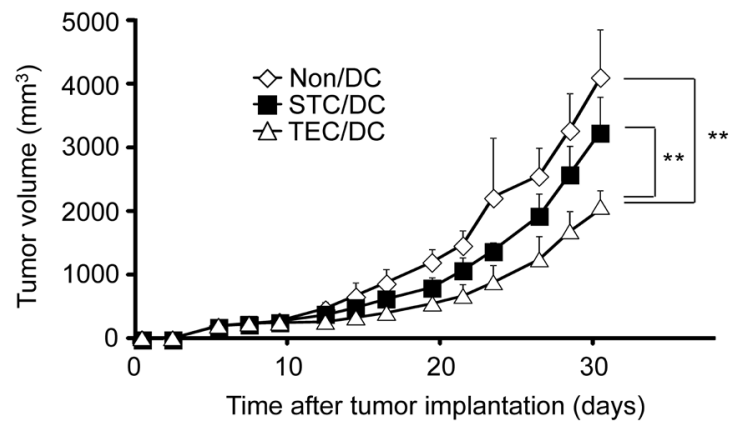

Fig. 2. Anti-tumor Effect of TEC- or Tumor Cell-Pulsed DC Vaccination in a Colon-26 Tumor-Bearing Mouse Model

Tumor growth curves of BALB/c mice implanted s.c. with $1 \times 10^{6}$ Colon-26 tumor cells in the left flank and vaccinated twice prophylactically in the right flank (days-14 and -7) (A, B), or vaccinated twice therapeutically in the right flank when the long diameter of tumor tissue reached $7 \mathrm{~mm}$ (days-14 and -7) (C). Tumor size was measured with a caliper. $* p<0.01$ or $* * p<0.001$ (One-way ANOVA). (A), DCs derived from $\mathrm{BALB} / \mathrm{c}$ mice were pulsed with Colon-26 tumor cell protein extracts (Colon-26/DC), TEC protein extracts from Colon-26 tumors (TEC/DC), or no antigen (Non/DC). ( $n=4$ per group). (B), Colon-26 solid tumor cell protein extracts (STC/DC), TEC protein extracts from Colon-26 tumors (TEC/DC), low ACE activity fraction in Colon-26 solid tumor tissue protein extract (Low ACE/DC; $n=6$ ), or without antigen (Non/DC; $n=5$ ), and with DCs used as adjuvant. (C), DCs were pulsed with Colon-26 solid tumor cell protein extracts (STC/DC), TEC protein extracts from Colon-26 tumors (TEC/DC), or without antigen (Non/DC). $(n=6$ per group). Values are expressed as mean \pm S.E.M.

abolished in TEC/DC-treated mice, an image similar to that seen for untreated mice without tumors $(p<0.001 v s$. Non/DC mice; Figs. 3C, A, D, respectively).

Moreover, histopathological analysis of tumor tissue revealed that many new blood vessels were recruited in Non/DC-treated mice (Figs. 4A, C). In contrast, it was observed that tumor blood vessels had decreased in solid tumors of TEC/DC-treated mice $(p<0.005$ vs. Non/DC mice; (Figs. $4 \mathrm{~B}, \mathrm{C})$ ). Thus, it is clear that vaccination using DCs pulsed with TECs inhibited angiogenesis in solid tumor tissues.

DC Vaccination with TECs Did Not Inhibit Physiological Angiogenesis To assess the influence of TEC/DC vaccina- 
A

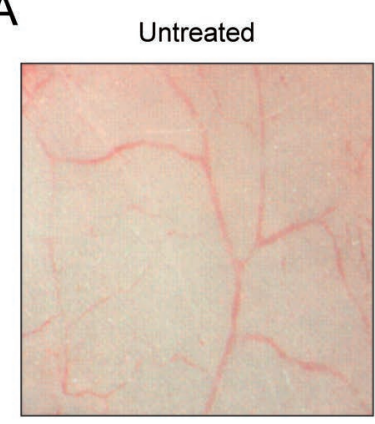

B

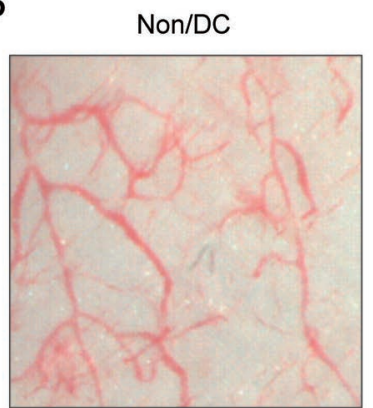

C

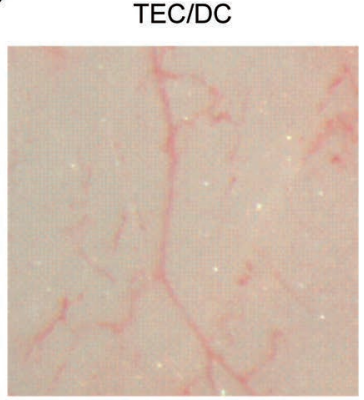

$\mathrm{D}$

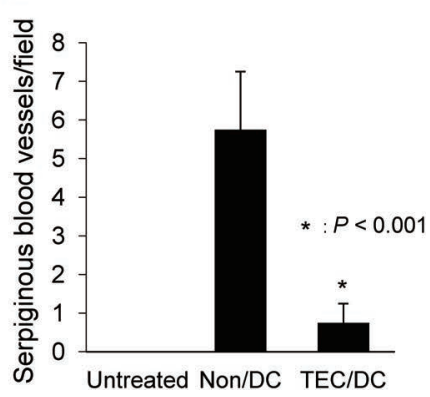

Fig. 3. Suppressive Effects of TEC/DC Vaccination on Tumor Angiogenesis in a Dorsal Air Sac Chamber Model

Each chamber was filled with a suspension of $1 \times 10^{6}$ Colon- 26 tumor cells and embedded s.c. into the backs of BALB/c mice. After $5 \mathrm{~d}$, each chamber was removed and observed by stereoscopic microscopy. (A), Unvaccinated mouse, which was embedded with a chamber without tumor cells (Untreated). (B, C), Mice were embedded with chambers filled with tumor cells, after being vaccinated prophylactically twice in the right flank with DCs derived from BALB/c mice (days-14 and -7). (B), DCs not pulsed with antigen (Non/DC). (C), DCs pulsed with TEC protein extracts from Colon-26 tumors (TEC/DC). Objective lens magnification: $(\times 16)$ Experiments were performed twice. (D) The total number of new blood vessels within the area attached by a chamber ring was assessed by averaging four random fields per specimen. Values are expressed as mean \pm S.E.M. ${ }^{*} p<0.001$ vs. Non/DC mice (one-way ANOVA).

tion on physiological angiogenesis, the time taken for wound healing in mice was measured (Fig. 5). In HSE/DC-vaccinated mice, a control for normal endothelial cells, a delay in recovery $(12.0 \pm 1.6 \mathrm{~d})$ from wound healing was observed, compared with that of Non/DC-vaccinated mice $(9.0 \pm 2.1 \mathrm{~d})$. In TEC/DCtreated mice, the time span for wound healing was only very slightly delayed $(9.6 \pm 1.9 \mathrm{~d})$ compared with that of Non/DCvaccinated mice. We confirmed that $\mathrm{DC}$ vaccination using HSE almost never inhibited B16 lung metastasis compared with untreated or Non/DC-vaccinated mice (data not shown). These results, therefore, suggest that vaccine therapy targeting TECs did not affect physiological angiogenesis.

DC Vaccination with TECs Derived from Different Types of Tumor Cell Lines Suppressed Lung Metastasis Tumor angiogenesis is prominently involved in tumor metastasis. Anti-metastatic effects by vaccination with DCs pulsed with TECs derived from different solid tumors were evaluated using a mouse lung metastasis model (Fig. 6). We ever confirmed that Non/DC vaccination hardly showed antimetastatic effect (data not shown). Vaccination with DCs exposed to TECs derived from B16 melanoma tumor tissue (B16 TEC/DC) significantly inhibited B16 lung metastases, whereas in the lungs of untreated mice, many metastatic colonies were observed $(p<0.001$ vs. untreated mice; Figs. 6A, B, C).

Moreover, to evaluate if TECs derived from a different type

A

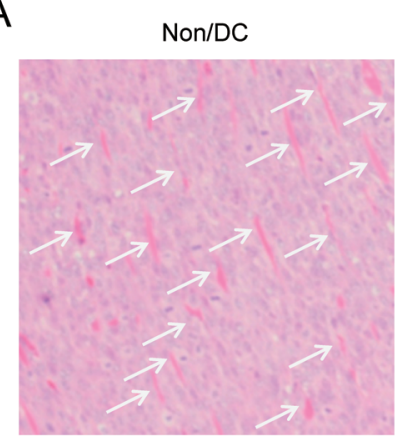

B

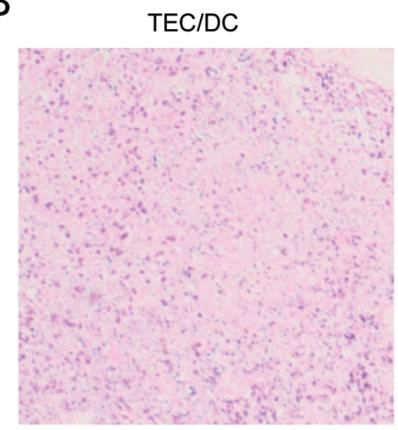

C

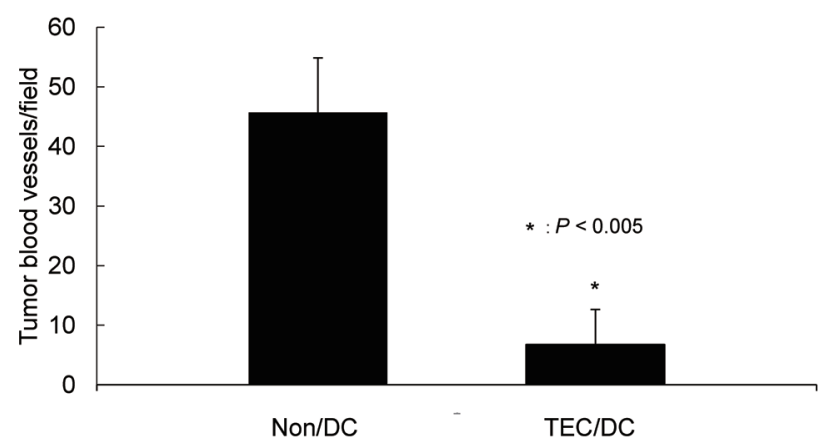

Fig. 4. Histopathological Analysis of the Anti-angiogenic Effects of TEC Vaccination

Tumor blood vessels formed in BALB/c mice implanted s.c. with $1 \times 10^{6}$ Colon- 26 tumor cells in the left flank. After $40 \mathrm{~d}$, Colon-26 solid tumor tissues were removed, stained with hematoxylin-eosin and observed by stereoscopic microscopy ( $n=3$ per group). Formation of new blood vessels (white arrows) in tumor tissues. Mice were twice vaccinated prophylactically in the right flank (days-14 and -7) (A) with BALB/c mice-derived DCs not pulsed with antigens (Non/DC), or (B) with DCs pulsed with TEC protein extracts from Colon-26 tumors (TEC/DC). Objective lens magnification: $(\times 100)$. (C) The total number of new blood vessels in the intratumoral section was assessed by averaging four random fields per specimen. Values are expressed as mean \pm S.E.M. ${ }^{*} p<0.005 v s$. Non/DC mice (Student's $t$-test).

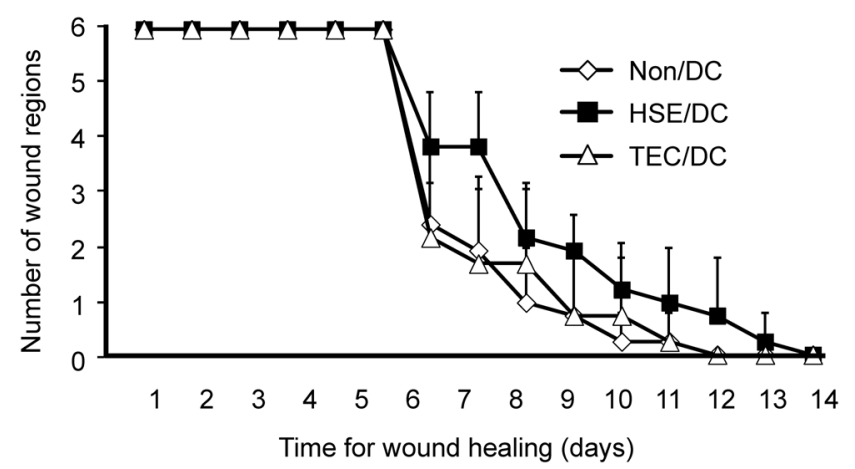

Fig. 5. Effect of TEC Vaccination on Angiogenesis in the Process of Wound Healing

Six circular wounds, each of $5 \mathrm{~mm}$ diameter, were made in the backs of BALB/c mice. Mice were vaccinated twice prophylactically in the right flank (days-14 and -7) with BALB/c mice-derived DCs pulsed with TEC protein extracts from Colon-26 tumor cells (TEC/DC), HSE cell (used as an endothelial cell control) protein extracts (HSE/DC) or no antigen (Non/DC). The number of wounds was counted and times until wound closure were measured ( $n=3$ per group). The average number of wounds per day. Values are expressed as mean \pm S.E.M.

of tumor cell line could also be useful as a therapeutic target for lung metastases, anti-metastatic effects induced by vaccination with DCs pulsed with TECs isolated from Colon-26 colorectal adenocarcinoma solid tumor tissues (Colon-26 TEC/DC) were also examined in the B16 mouse lung metas- 
A

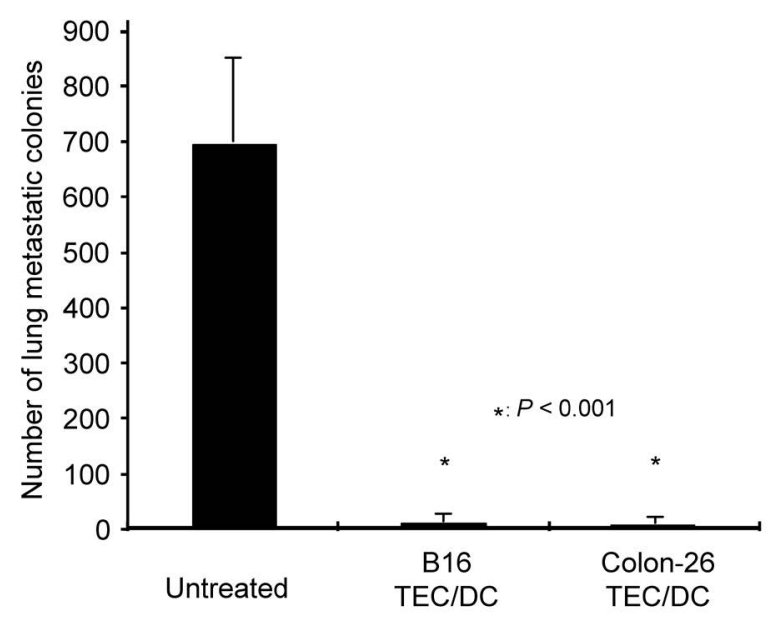

$\mathrm{B}$

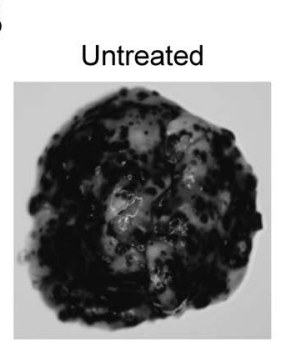

C

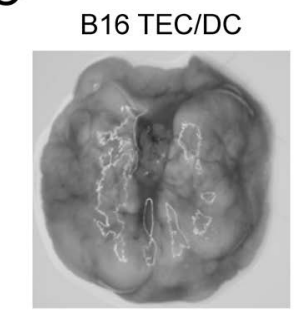

D

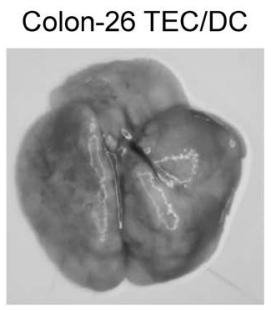

Fig. 6. Anti-metastatic Effects of TEC Vaccination in a B16 Lung Metastasis Model

B16 cells $\left(1.0 \times 10^{5}\right)$ were injected i.v. in the tail vein of C57BL/6 mice, which had been prophylactically injected twice in the right flank (days-14 and -7$)$ with C57BL/6 mice derived-DCs pulsed with TEC protein extracts isolated from a B16 tumor (B16 TEC/DC; $n=6)$, with TEC protein extracts isolated from a Colon-26 tumor (Colon-26 TEC/DC; $n=5$ ), or had not been injected (untreated; $n=7$ ). (A), Fourteen days after a B16 cell injection, B16 cell colonies in lung tissues were counted. Values are expressed as mean \pm S.E.M. ${ }^{*} p<0.001 v s$. untreated mice (one-way ANOVA). (B), Lungs from untreated, (C), B16 TEC/DC-, and (D), Colon-26 TEC/DC-treated mice.

tasis model (Figs. 6A, D). In Colon-26 TEC/DC-treated mice, lung metastases were significantly suppressed in a manner similar to B16 TEC/DC-treated mice $(p<0.001$ vs. untreated mice; Figs. 6A, C, D). Thus, TECs isolated from at least two different solid tumor tissues showed potential as therapeutic targets for lung metastases, regardless of whether TECs were isolated from the existing or another tumor type.

\section{DISCUSSION}

We hypothesized that TECs may be useful as target cells for vaccination in cancer therapy since tumor blood vessels play a predominant role in tumor growth and metastasis. However, methods to isolate and culture TECs from tumor tissues are not yet well established since specific marker molecules of TECs have not been identified. In recent years, tumor endothelial marker 8 (TEM8) and aminopeptidase N (APN/ CD13) have been discovered to be expressed predominantly in TECs. ${ }^{24,25)}$ Additionally, these molecules were barely detectable in endothelial cells, or in the proliferative endothelium of the corpus luteum or healing wounds. ${ }^{26,27)}$ Therefore, DC vaccine therapy using TECs as antigen has not been successfully attempted.

Herein, we describe the isolation of TECs, by density gradient centrifugation, from cell fractions derived from tumor tissues. TEC-rich fractions were subsequently identified on the basis of ACE activities, and which also displayed high CD34 expression on cell surfaces.

Next, to elucidate the utility of isolated TECs as TAAs, their anti-tumor effects in DC vaccination were evaluated. As a result, we found that vaccination with DCs pulsed with TECs could efficiently induce tumor regression, compared with that of tumor cells themselves. Moreover, DC vaccination using solid tumor extracts containing almost no TECs did not suppress tumor progression. These results suggested that tumor growth inhibition by DC vaccination using TECs was induced by TAAs associated with TECs that displayed high ACE activities. Additionally, in terms of therapeutic administration, DC vaccination using TECs also succeeded in strongly inhibiting tumor progression. Therefore, it is postulated that DC vaccination targeting TECs will induce anti-tumor effects, even after tumor-induced angiogenesis is well established. In TEC/DC vaccine-treated mice, serpiginous new vessels allowing blood flow to tumor tissues during tumor growth disappeared completely compared with those of DC only vaccinated mice. It was shown that the anti-tumor effects of vaccine therapy targeting TECs were induced by the suppression of new blood vessel formation. Thus, TECs contained within solid tumor tissues can be an effective therapeutic target in cancer therapy for solid tumors.

Moreover, overcoming tumor metastasis is a high-priority in cancer therapy. For example, in a mouse lung metastasis model, DC vaccination using TECs derived from solid tumors strongly suppressed lung metastasis compared with untreated 
mice. Interestingly, vaccination using TECs isolated from a different solid tumor type showed the same anti-metastatic effects as that of TECs derived from the same solid tumor, thus suggesting that vaccine therapy with TECs can suppress metastasis in different types of cancer. Recently, other investigators have reported that DNA vaccination based on the TEM8 gene using DCs as adjuvant resulted in the deceleration of tumor growth by enhancing anti-angiogenic effects via the induction of TEM8-specific CTLs and interferon- $\gamma$ production; the effects decreased after the depletion of CD8-positive $\mathrm{T}$ cells. ${ }^{28)}$ Moreover, DC vaccination using adenovirus that expressed both telomerase reverse transcriptase and VEGF receptor 2 (VEGFR2) pointed to the inhibition of tumor angiogenesis by activating VEGFR2-specific CTLs. ${ }^{29)}$ Additionally, our previous study showed that normal endothelial cells cultured in tumor conditioned medium (in vitro TEC model) enhanced the permeabilization of the endothelial cell monolayer to molecules compared with those cells cultured in normal medium, in common with the characteristics of tumor blood vessels in vivo. ${ }^{30)}$ Also, DC vaccination using in vitro TEC model protein extracts as antigens suppressed lung metastasis significantly via the destruction of tumor blood vessels by inducing TEC-specific CTLs as shown in an in vitro cytotoxicity assay. Anti-metastatic effects were inhibited by the administration of anti-CD8 or -CD4 monoclonal antibody (data not shown). In this study, although the mechanism of immune induction by DC vaccination using isolated TECs was not analyzed in detail, the above report suggests that our cancer vaccine therapy induced immunity for TEC-specific antigens and showed anti-tumor effects by damaging tumor blood vessels.

Besides pathological tumor growth, angiogenesis also occurs under physiological conditions, such as in wound healing and in the formation of the corpus luteum. To develop a vaccination protocol targeting TECs as a cancer therapy without side effects, it is important that such therapy does not affect physiological angiogenesis. In this regard, vaccination with normal endothelial blood vessel cells (HSE/DC) as a negative control tended to delay wound healing, compared with solely DC vaccination, indicating the formation of new blood vessels was uniformly suppressed. In contrast, in TEC/DC vaccine-treated mice, wound healing was only marginally delayed, with the assumption being this was due to tumor blood vessel-selective damage by TEC vaccination. Vaccine therapy using targeted molecules, such as tumor endothelial marker 1, TEM8, fibroblast growth factor receptor or VEGFR2, which are overexpressed on TECs, has been studied by many investigators. $^{31-34)}$ However, a TEC-specific vaccine therapy with few side-effects has not been developed as yet since such molecules are also expressed on the cell surface of normal blood vessel endothelial cells. In this regard, our results strongly suggest that tumor vaccine therapy only targets tumor, but not physiological, angiogenesis.

\section{CONCLUSION}

Cancer therapy using existing anti-angiogenic agents, such as tyrosine-kinase inhibitors and anti-VEGF antibodies, are used clinically for cancer patients to successfully prolong their survival. However, such drugs have known side effects such as hypertension and thrombotic disease, since these drugs also inhibit VEGF/VEGF receptor signaling in normal blood vessels. Moreover, these drugs are limited to the applicable types of tumor. In this regard, our study suggests that TECs in several types of tumor tissues can express common, TECspecific TAAs that are not expressed on normal endothelial cells. Therefore, our therapeutic strategy is an effective cancer immunotherapy against several types of tumor tissues without side effects. In future, the precise identification of specific markers in TECs is expected to lead to the development of increasingly targeted cancer therapies, such as TEC-specific antibodies and vaccines for solid tumors or metastases, as viable alternatives to existing anti-angiogenic agents.

Acknowledgments The authors thank Drs. S. Unezaki, and I. Saiki for providing materials. This work was supported in part by the Japan Society for the Promotion of Science (JSPS) KAKENHI Grant Number (16790110), and the Ministry of Education, Culture, Sports, Science and Technology (MEXT)-Supported Program for the Strategic Research Foundation at Private Universities 2013-2017.

Conflict of Interest The authors declare no conflict of interest.

\section{REFERENCES}

1) Lyden D, Hattori K, Dias S, Costa C, Blaikie P, Butros L, Chadburn A, Heissig B, Marks W, Witte L, Wu Y, Hicklin D, Zhu Z, Hackett NR, Crystal RG, Moore MA, Hajjar KA, Manova K, Benezra R, Rafii S. Impaired recruitment of bone-marrow-derived endothelial and hematopoietic precursor cells blocks tumor angiogenesis and growth. Nat. Med., 7, 1194-1201 (2001).

2) Folkman J, Watson K, Ingber D, Hanahan D. Induction of angiogenesis during the transition from hyperplasia to neoplasia. Nature, 339, 58-61 (1989).

3) Kim KJ, Li B, Winer J, Armanini M, Gillett N, Phillips HS, Ferrara N. Inhibition of vascular endothelial growth factor-induced angiogenesis suppresses tumour growth in vivo. Nature, 362, 841-844 (1993).

4) Folkman J, Klagsbrun M. Angiogenic factors. Science, 235, 442 447 (1987).

5) Li H, Fredriksson L, Li X, Eriksson U. PDGF-D is a potent transforming and angiogenic growth factor. Oncogene, 22, 1501-1510 (2003).

6) Gupta MK, Qin RY. Mechanism and its regulation of tumor-induced angiogenesis. World J. Gastroenterol., 9, 1144-1155 (2003).

7) Folkman J. The role of angiogenesis in tumor growth. Semin. Cancer Biol., 3, 65-71 (1992).

8) Carmeliet P. Mechanisms of angiogenesis and arteriogenesis. Nat. Med., 6, 389-395 (2000).

9) Atkins M, Jones CA, Kirkpatrick P. Sunitinib maleate. Nat. Rev. Drug Discov., 5, 279-280 (2006).

10) Yang JC. Bevacizumab for patients with metastatic renal cancer: an update. Clinical Cancer Research: An Official Journal of the American Association for Cancer Research, 10, 6367S-6370S (2004).

11) Ferrara N, Chen H, Davis-Smyth T, Gerber HP, Nguyen $T N$, Peers D, Chisholm V, Hillan KJ, Schwall RH. Vascular endothelial growth factor is essential for corpus luteum angiogenesis. Nat. Med., 4, 336-340 (1998).

12) Clark AJ, Butowski NA, Chang SM, Prados MD, Clarke J, Polley MY, Sughrue ME, McDermott MW, Parsa AT, Berger MS, Aghi MK. Impact of bevacizumab chemotherapy on craniotomy wound healing. J. Neurosurg., 114, 1609-1616 (2011).

13) Mody N, Dubey S, Sharma R, Agrawal U, Vyas SP. Dendritic cell- 
based vaccine research against cancer. Expert Rev. Clin. Immunol., 11, 213-232 (2015).

14) Banchereau J, Steinman RM. Dendritic cells and the control of immunity. Nature, 392, 245-252 (1998).

15) Pamer E, Cresswell P. Mechanisms of MHC class I-restricted antigen processing. Annu. Rev. Immunol., 16, 323-358 (1998).

16) Evel-Kabler K, Chen SY. Dendritic cell-based tumor vaccines and antigen presentation attenuators. Mol. Ther., 13, 850-858 (2006).

17) Takahashi H, Okamoto M, Shimodaira S, Tsujitani S, Nagaya M, Ishidao T, Kishimoto J, Yonemitsu Y. Impact of dendritic cell vaccines pulsed with Wilms' tumour-1 peptide antigen on the survival of patients with advanced non-small cell lung cancers. Eur. J. Cancer, 49, 852-859 (2013).

18) Chen YT, Scanlan MJ, Sahin U, Tureci O, Gure AO, Tsang S, Williamson B, Stockert E, Pfreundschuh M, Old LJ. A testicular antigen aberrantly expressed in human cancers detected by autologous antibody screening. Proc. Natl. Acad. Sci. U.S.A., 94, 1914-1918 (1997).

19) Lepisto AJ, Moser AJ, Zeh H, Lee K, Bartlett D, McKolanis JR, Geller BA, Schmotzer A, Potter DP, Whiteside T, Finn OJ, Ramanathan RK. A phase I/II study of a MUC1 peptide pulsed autologous dendritic cell vaccine as adjuvant therapy in patients with resected pancreatic and biliary tumors. Cancer Ther., 6 (B), 955-964 (2008).

20) Utoguchi N, Dantakean A, Makimoto H, Wakai Y, Tsutsumi $Y$, Nakagawa S, Mayumi T. Isolation and properties of tumor-derived endothelial cells from rat KMT-17 fibrosarcoma. Jpn. J. Cancer Res., 86, 193-201 (1995).

21) Inaba $\mathrm{K}$, Inaba $\mathrm{M}$, Romani $\mathrm{N}$, Aya $\mathrm{H}$, Deguchi $\mathrm{M}$, Ikehara $\mathrm{S}, \mathrm{Mu}-$ ramatsu S, Steinman RM. Generation of large numbers of dendritic cells from mouse bone marrow cultures supplemented with granulocyte/macrophage colony-stimulating factor. J. Exp. Med., 176, 1693-1702 (1992).

22) Hasumi Y, Mizukami H, Urabe M, Kohno T, Takeuchi K, Kume A, Momoeda M, Yoshikawa H, Tsuruo T, Shibuya M, Taketani Y, Ozawa K. Soluble FLT-1 expression suppresses carcinomatous ascites in nude mice bearing ovarian cancer. Cancer Res., 62, 2019-2023 (2002).

23) Mabuchi S, Terai $Y$, Morishige $K$, Tanabe-Kimura A, Sasaki H, Kanemura M, Tsunetoh S, Tanaka Y, Sakata M, Burger RA, Kimura T, Ohmichi M. Maintenance treatment with bevacizumab prolongs survival in an in vivo ovarian cancer model. Clinical Cancer Research: An Official Journal of the American Association for Cancer Research, 14, 7781-7789 (2008).

24) Carson-Walter EB, Watkins DN, Nanda A, Vogelstein B, Kinzler KW, St Croix B. Cell surface tumor endothelial markers are con- served in mice and humans. Cancer Res., 61, 6649-6655 (2001).

25) Fukasawa K, Fujii H, Saitoh Y, Koizumi K, Aozuka Y, Sekine K, Yamada M, Saiki I, Nishikawa K. Aminopeptidase N. APN/CD13) is selectively expressed in vascular endothelial cells and plays multiple roles in angiogenesis. Cancer Lett., 243, 135-143 (2006).

26) Wang SC, Ye L, Sanders AJ, Ruge F, Harding KG, Jiang WG. Tumour endothelial marker- 8 in wound healing and its impact on the proliferation and migration of keratinocytes. Int. J. Mol. Med., 37, 293-298 (2016).

27) Rahman MM, Ghosh M, Subramani J, Fong GH, Carlson ME, Shapiro LH. CD13 regulates anchorage and differentiation of the skeletal muscle satellite stem cell population in ischemic injury. Stem Cells, 32, 1564-1577 (2014).

28) Liu P, Xie G, Geng P, Zheng C, Li J, Pan F, Ruan Z, Liang H. Anti-tumor angiogenesis effect of genetic fusion vaccine encoding murine beta-defensin 2 and tumor endothelial marker- 8 in a CT-26 murine colorectal carcinoma model. International Journal of Clinical and Experimental Medicine, 8, 4744-4752 (2015).

29) Wang Y, Zhang J, Wu Y, Ding ZY, Luo XM, Liu J, Zhong WN, Deng GH, Xia XY, Deng YT, Wei YQ, Jiang Y. Mannan-modified adenovirus targeting TERT and VEGFR-2: A universal tumour vaccine. Scientific Reports, 5, 11275 (2015).

30) Utoguchi N, Mizuguchi H, Saeki K, Ikeda K, Tsutsumi Y, Nakagawa S, Mayumi T. Tumor-conditioned medium increases macromolecular permeability of endothelial cell monolayer. Cancer Lett., 89, 7-14 (1995)

31) Facciponte JG, Ugel S, De Sanctis F, Li C, Wang L, Nair G, Sehgal S, Raj A, Matthaiou E, Coukos G, Facciabene A. Tumor endothelial marker 1-specific DNA vaccination targets tumor vasculature. $J$. Clin. Invest., 124, 1497-1511 (2014).

32) Yang $\mathrm{X}, \mathrm{Zhu} \mathrm{H}, \mathrm{Hu} \mathrm{Z}$. Dendritic cells transduced with TEM8 recombinant adenovirus prevents hepatocellular carcinoma angiogenesis and inhibits cells growth. Vaccine, 28, 7130-7135 (2010).

33) He QM, Wei YQ, Tian L, Zhao X, Su JM, Yang L, Lu Y, Kan B, Lou YY, Huang MJ, Xiao F, Liu JY, Hu B, Luo F, Jiang Y, Wen YJ, Deng HX, Li J, Niu T, Yang JL. Inhibition of tumor growth with a vaccine based on xenogeneic homologous fibroblast growth factor receptor-1 in mice. J. Biol. Chem., 278, 21831-21836 (2003).

34) Masuzawa T, Fujiwara Y, Okada K, Nakamura A, Takiguchi S, Nakajima K, Miyata H, Yamasaki M, Kurokawa Y, Osawa R, Takeda K, Yoshida K, Tsunoda T, Nakamura Y, Mori M, Doki Y. Phase I/II study of S-1 plus cisplatin combined with peptide vaccines for human vascular endothelial growth factor receptor 1 and 2 in patients with advanced gastric cancer. Int. J. Oncol., 41, 1297-1304 (2012). 